\title{
An Empirical Inquiry into the Perceptual Qualities of Spatial Enclosures in Head Mounted Display Driven VR Systems
}

\section{Quantifying the 'Intangibles' of Space}

\author{
Rohit Priyadarshi Sanatani ${ }^{1}$ \\ ${ }^{1}$ CEPT University \\ ${ }^{1}$ rohitsanatani@yahoo.co.in
}

\begin{abstract}
This research is an inquiry into correlations between specific configurations of virtual spatial enclosures and corresponding perceptual responses in subjects. The experiments comprised of three sets - opening configurations, volume configurations and partition configurations. The perceptual parameters tested were Degree of Enclosure (E), Degree of Separation (P), and Spaciousness (S) respectively. Immersive virtual environments depicting enclosures with these different configurations were presented to 25 subjects through a head mounted $V R$ gear. Responses were recorded in the form of verbal ratings. The results revealed that one's visual field along the horizontal axis at eye level plays a major role in the way specific attributes of spatial enclosures are perceived. One's perception of 'openness' in an enclosure correlated strongly with the amount of physical opening that was present along the horizontal axis at eye level, while the perception of 'spaciousness' correlated strongly with the amount of visual obstruction within one's horizontal visual field at eye level. It was found that larger unified openings between enclosures along eye level created a weaker sense of visual separation as compared smaller dispersed openings of equal cumulative area.
\end{abstract}

\section{INTRODUCTION}

It is well known that specific attributes of spatial enclosures directly lead to them to being perceived in very specific ways. We know intuitively, for example, that a room comprising of a number of volumetric elements (furniture etc) 'appears' to be smaller than the same room without such visual interruptions. The effect of perception, on our understating and engagement with space is something that we have all intuitively experienced.
The 'perceptual' qualities of spatial enclosures can be said to lie within the realm of spatial 'experience'. This realm, despite occupying a central position in the domain of architecture and design, has long been regarded as a collection of 'intangibles', which cannot be objectively measured. It is only in the recent past, that the scientific method has been applied to the realm of experience in space, guided by the broad hypothesis that specific aspects and configurations of spatial enclosures result in them 
being perceived in very specific ways. There is still not a great deal of research that specifically inquires into this domain.

A focused study empirically inquiring into the correlations between spatial configuration and perception has immense potential to yield valuable lines of enquiry within the realm of spatial experience. More importantly, data derived from such research can provide designers with logical grounding with respect to design decisions taken keeping the realm of experience in mind.

Over the past few decades, the development of immersive, responsive and interactive virtual representations of space, which are capable of generating simulations that can approximate the sensory inputs provided by a real space, has immense potential to develop into a valuable tool for such lines of empirical research. Recent developments in virtual environment simulations include Head Mounted Displays fitted with audio output and a gyroscope. The stereoscopic visual output is done through and inbuilt OLED display and a pair of convex lenses. The gyroscope and accelerometer senses head position and orientation and changes the display scene accordingly. (Mazuryk et al 2010) A number of manufacturers such as Oculus and Sony have in recent years introduced a range of wearable HMDs. While VR systems in various stages of development have been applied in a few similar studies in the past (Franz et al 2003) (Shemesh et al 2015), it is the rapid development in the field of VR in recent years, and the widespread commercial production of head mounted display (HMD) devices that have made the use of such systems for architectural research readily accessible.

\section{SELECTED PERCEPTUAL ATTRIBUTES FOR EXPERIMENTATION}

While there are an incalculable number of factors that determine the way one perceives a space, Bitner and Schachter identified three primary categories of attributes that influence the experience of individuals inhabiting it, namely: (i) Formal attributes, (ii) Signs, symbols and artifacts, and (iii) Ambient conditions (Bitner 1992)

This body of research has restricted itself to the first category. There are a number of primary attributes of form, namely shape, size, color, texture, position, orientation and visual inertia. (Ching 1996) The experiments conducted as a part of this study was directed towards recording specific perceptual attributes of different architectural configurations, namely Degree of Enclosure (E), Degree of Separation (P), and Spaciousness (S). The architectural configuration sets selected for studying these attributes were comprised of discrete formal configurations, which were commonly found in architectural practice. The experiments comprised of 3 configuration sets specifically focused towards studying responses pertaining to the 3 perceptual attributes.

\section{Opening Configurations}

The first set, studying Degree of Enclosure (E) comprised of five enclosures of fixed area of opening (5.25 sqm), but different opening configurations as shown in (Figure 1). The configurations included the following: (1) horizontal window, (2) vertical window, (3) skylight, (4) split vertical window, and (5) split corner window. For each of the instances, subjects were asked to rate their perceived degree of enclosure on a scale of 0 to 5 . The specific underling hypothesis behind the experiment was that despite an equal area of opening, different opening configurations correlated with different 'senses of enclosure' in a space. The experiment was focused towards understanding the nature of such correlations.

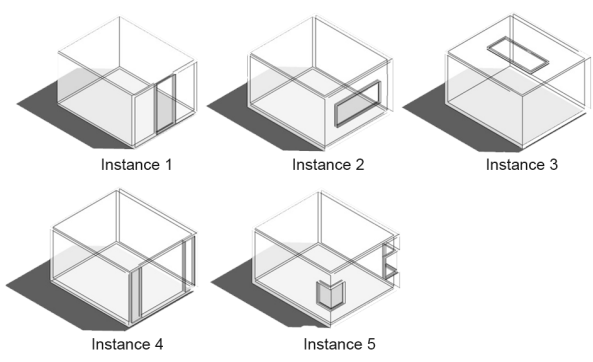

Figure 1

Configuration Set 1:

Opening Configurations 
Figure 2

Configuration Set 2:

Partition

Configurations

Figure 3

Configuration Set 3:

Volume

Configurations

Figure 4

360 degree

spherical renders

for VR viewing

\section{Partition Configurations}

The second set looked into the perceptual attribute of Degree of Separation (P). Each of the instances comprised of two adjacent enclosures of equal size separated by a partition. The five instances used in the experiment varied according to the configuration of the partition separating the spaces. (Figure 2) The partition configurations were (1) Wall opening (2) Dense Bars (3) Broad Bars and (4) Low height wall with clerestory opening. The total area of physical opening between the spaces that each of these partitions allowed, was constant (7 sq.m). The subjects were asked to rate their perceived degree of separation for each of these spaces. The hypothesis was that different partition configurations of same opening area produce different degrees of 'perceived separation' in occupants.

\section{Volume Configurations}

The third set of spaces used for the experiments were directed towards studying the varying degrees of Perceived Spaciousness (S) that different configurations of enclosures of same volume gave rise to. The logic behind the volume configurations was the subtractive transformation of form. Each of the enclosures comprised of a rectangular volume with another smaller rectangular volume subtracted from it. This was done twice in the form of toilets and once in the form of a loft. (Figure 3) The absolute value of volume subtracted, however remained the same for all the variants (10.5 cu.m). The variants were (1) Loft, (2) Corner Toilet and (3) Center Toilet. Even though enclosure may undergo subtractive transformation in a number of ways, these were found to be the most commonly occurring cases in mainstream architectural practice. Since the absolute value of enclosure volume remained the same for all variants, the effect of the partition configurations on perceived spaciousness could be accurately tested. The subjects were asked to attribute a value of spaciousness for each of these spaces.
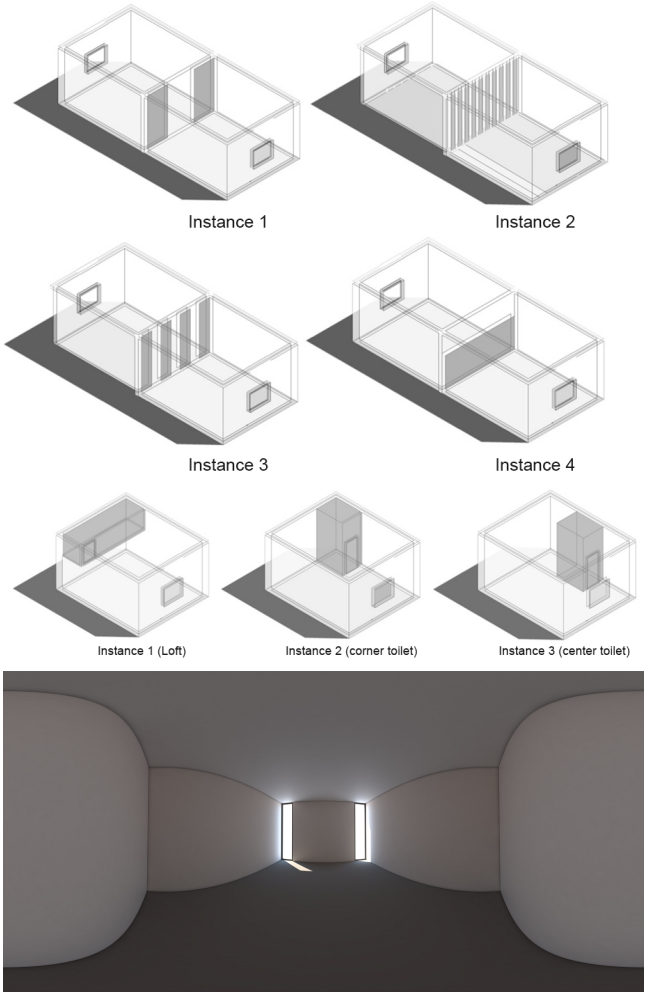

\section{METHODOLOGY}

\section{Generating immersive virtual environ- ments}

Each of these enclosures was modelled on Google SketchUp and rendered using VRay for SketchUp. The final images were spherical renders of field of view (FOV) $360^{\circ}$ and resolution $4000 \times 2000$ pixels. (Figure 4). The camera height was uniformly maintained at $1500 \mathrm{~mm}$ above floor level. A Fulldrive VR engine adapted these spherical renders for $360^{\circ}$ display through the VR gear. 


\section{Gear and Setup}

The VR gear comprised of a Head Mounted Device (HMD) comprising of a VR Viewer and the Display Unit. The display unit was an android driven mobile device (Lenovo Vibe K5 Note) with a diagonal display dimension of 5.5 inches and a display resolution of 1080 pixels. This unit was fitted inside the VR Viewer, which was a Procus One unit with biconvex lenses of focal length $14 \mathrm{~mm}$. The inter-pupillary distance and distance between the display surface and lenses could both be adjusted by the subjects through knob provided on the VR unit. The display that was being shown to the subject at any point of time was streamed live to a laptop (MacBook Pro 8.1) via a steaming engine (Mirror Beta). The immersive spatial environments in the form of spherical renders were adapted and displayed through an android driven VR engine (Fulldrive).

\section{Synthesis and Rating of Perceptual Param- eters}

A number of scales have been devised in the past to objectively synthesise perceptual parameters of space. Scales such as the SMB scale employ parameters such as pleasantness, complexity, unity or coherence, enclosedness, potency, social status, affection, and originality (Küller 1972). For the selected formal attributes, however, very focused perceptual parameters needed to be synthesised, so as to maximise relevance to mainstream architectural practice and intuitive architectural experience. The 3 perceptual attributes namely Degree of Enclosure (E), Degree of Separation (P), and Spaciousness $(S)$ were thus selected, and the subjects were asked to verbally rate the respective parameters on a 0 - 5 scale (similar to affective appraisals). (Houtkamp 2012)

\section{Sampling}

The experiments comprised of a sample of $(\mathrm{N}=25)$ subjects. The secondary experiment allowing movement within the space for the spaciousness parameter had a sample of $(\mathrm{N}=5)$ subjects. All subjects for all experiments were postgraduate students of CEPT University, Ahmedabad, within the age group $23-33$, with a bachelor's degree in Architecture.

\section{RESULTS AND DISCUSSION}

The results were fairly consistent across all subjects, with very few instances of deviation from the established patterns. Analysis of the data obtained has yielded a number of valuable inferences, which give us a critical insight into the perceptual aspects of architectural experience. Let us consider the inferences from the three parameter-sets separately.

\section{Opening configurations and perceived de- gree of enclosure}

Let us begin with the set that studied the correlations between different configurations of window openings and perceived degrees of enclosure (E). We have seen from the results (Figure 5 ) that instance 2, i.e. a horizontal window opening corresponded with the lowest rated degree of enclosure, while the skylight (instance 3) corresponded to the lowest rated value. General qualitative responses from subjects during experimentation suggested that the room with the skylight appeared most enclosed because they could not find any opening at eye level. Since all the subject station points were placed at the far end of the enclosure, near the wall opposite the window openings, the skylight configuration corresponded to a situation where the subjects were physically nearest to an external opening (due to the fact the skylight was an opening on the ceiling plane). However despite this fact, the skylight configuration corresponded to the highest degree of rated enclosure because there was no opening on the horizontal plane the average eye level.

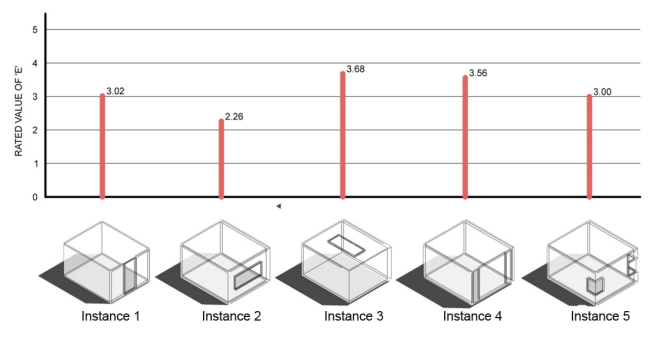

Figure 5

Rated degree of Enclosure (E) for different opening configurations 
Figure 6

Correlation of rated ' $E$ ' with the amount of external opening along the horizontal axis at eye level within the field of view of the subject
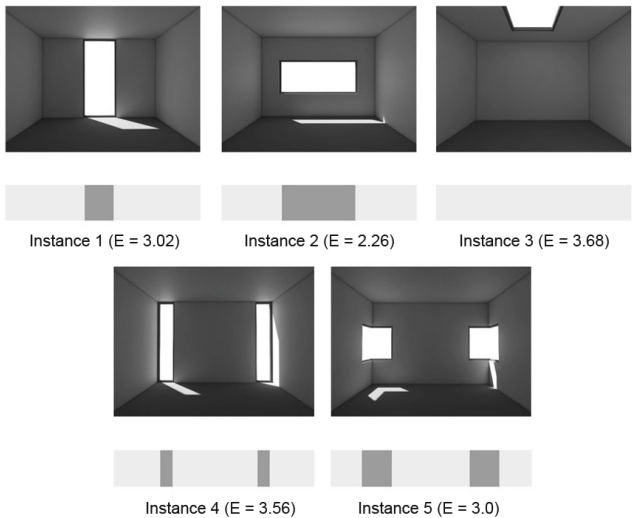

A closer analysis of the data revealed something more interesting. The issue of openings at eye level did not remain restricted to the enclosure with the skylight. There was a strong underlying pattern behind the way subjects responded to the spaces. The data shows that the rated degree of enclosure for each of the variants directly correlated with the amount of external opening along the horizontal axis at eye level within the field of view of the subject. (Figure 6) shows a diagrammatic representation of the horizontal openings at eye level, along with the rated values of degree of enclosure. It is evident, that as the amount of opening along the horizontal axis increases, the rated degree of enclosure decreases. This rule holds true for all variants. Instance 2 , being a horizontal window thus has the greatest amount of opening along the horizontal axis at eye level, and thus corresponds to the lowest value of perceived degree of enclosure.

The large degree to which horizontal openings at eye level influence our perception of enclosure gives us a very valuable insight into possible design directions. The knowledge of this factor can be turned to our advantage while trying to impart a sense of 'openness' to an enclosure during the process of design. It can also caution us against using architectural devices that appear to impart e sense of openness, but actually do so to a very small degree. Open- ness and enclosure are important tools that architects employ during design. A greater degree of enclosure usually imparts a greater degree of privacy, while a greater sense of openness provides a greater connection with the outside world, thus reducing the sense of privacy. It is evident from the results that total opening size and perceived openness, though strongly related, are not necessarily related exclusively. The dominant effect of openings at eye level can thus be used in the architects' favor in sensitive design decisions. Opening placement can be maximized along horizontal eye level, to impart a far greater degree of openness for a fixed area of opening.

\section{Volume configurations and perceived spa- ciousness.}

The experiment studying the effect of different volume configurations on the perceived spaciousness of an enclosure also yielded some valuable inferences that can be utilized during the design process. As described in the methodology section, a fixed orthogonal volume of space was subtracted from an enclosure, twice in the form of toilets, and one in the form of a loft. The data reveals that the configuration with the loft corresponded to the highest degree of rated spaciousness, while the configuration with the toilet at the center of one side corresponded to the lowest degree of rated spaciousness. (Figure 7) Qualitative responses during experimentation revealed that the center toilet appeared to block the subjects' view of the enclosure in its entirety, thus giving a lower perception of spaciousness. Both the corner toilet and loft allowed for the viewing of the entire space. The loft, however, corresponded to a much higher score on perceived spaciousness as compared to the corner toilet. Running responses revealed that the loft, being at a higher level, did not appear to diminish the sense of space as much as the corner toilet did.

Critical analysis revealed an underlying pattern that was in a way similar to the inferences derived from the previous set of opening configurations. It was found that the degree of rated spaciousness ap- 
peared to correlate strongly with the amount of visual obstruction existing within the subjects' visual field along the horizontal axis at eye level. Shown below (Figure 8 ) is a diagrammatic representation of the degree to which each of the volume configurations presented obstructions within the subjects' visual field along the horizontal axis at eye level.

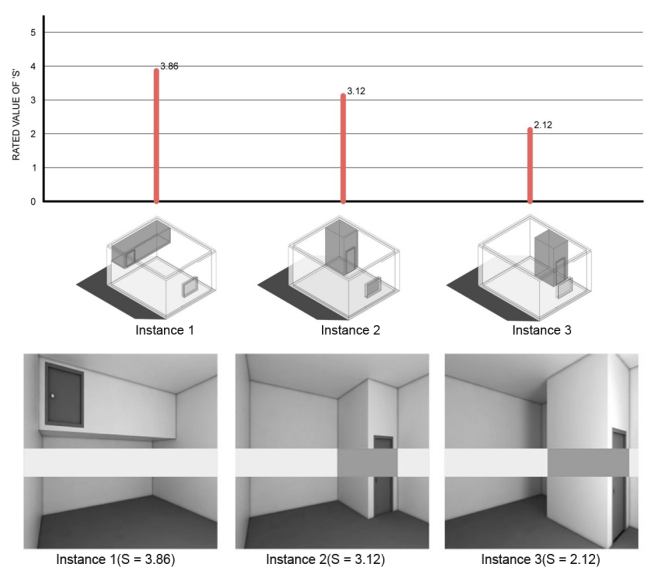

For all the variants, the station point was fixed at the corner diagonally opposite the corner with the toilet. The center toilet presents the maximum obstruction, and thus corresponds to the lowest value of rated spaciousness. The corner toilet, being farther away presents a lower degree of obstruction and thus corresponds to a higher value of spaciousness. The rated value of spaciousness for the loft is highest because it does not present any visual obstruction at average eye height.

One factor that must be taken into account for this set is the effect of station point on perceived spaciousness. Since the position from which one view the enclosure defines his or her visual field, that same element (say toilet) may present different degrees of obstruction depending on the position of the observer. This would in turn determine the degree of perceived spaciousness in that enclosure. To overcome the restrictions imposed by station points, it thus became necessary to carry out a control ex- periment where subjects would be allowed to move around and explore the space from different station points before giving his or her response.

This control experiment was carried out as described in the methodology section with a small sample size of $\mathrm{N}=10$ people. The subjects were allowed to move around using a handheld remote device. The results obtained from this experiment were very marginally different from the main experiment, with the relative order of perceived spaciousness remaining the same between the three variants. (Figure 9) This goes on to show that movement did not play a very major role in the way subjects perceived spaciousness. One major reason behind this could have been the fact that, despite the changing visual fields, the center toilet continued to present maximum visual obstruction from most station points, while the loft, being well above eye level, did not present any obstruction.

An insight into the way we perceive spaciousness has the potential to inform design decisions to a great degree. Physical space and perceived spaciousness are related entities, but not the same. The larger a physical space is in absolute terms does directly imply a greater degree of perceived spaciousness. All the spaces tested in this set yielded varying degrees of rated spaciousness for a fixed value of physical volume. As designers, it is perceived spaciousness and not necessarily physical space that we often want to maximize. As this set of experiments demonstrate, the relations between space and spaciousness is far more complex, and involves a number of perceptual factors.

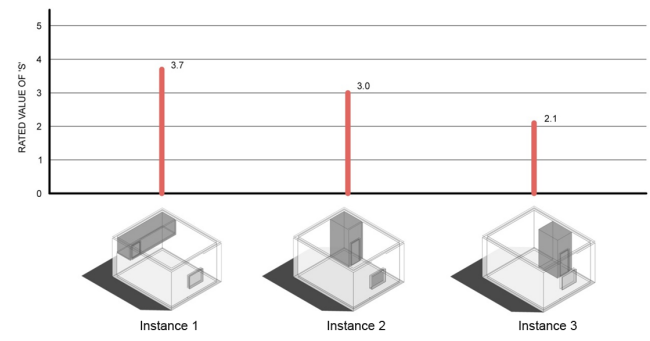

Figure 7

Rated Spaciousness

(S) for different

volume

configurations

Figure 8

Correlation of rated ' $S$ ' with the amount of visual obstruction along the horizontal axis at eye level within the field of view of the subject

Figure 9

Rated Spaciousness

(S) for different

volume

configurations

(Control experiment with movement) 
Figure 10

Rated degree of Separation (P) for different partition configurations

Figure 11

Larger, unified

openings along

horizontal axis at eye level correspond to lower values of rated ' $\mathrm{P}$ '
The relation inferred through this study can be used to the advantage of designers in a multitude of ways. Rather than approaching the maximization of spaciousness purely through the maximization of physical space, one can, over and above that, approach it through the minimization of visual obstruction at eye level. Smaller volumes within large enclosures commonly occur as toilets, lofts, closets, or arise due to the intersection of two adjacent enclosure volumes. Different geometric configurations for the same absolute volume of enclosure also present very different degrees of visual obstruction with respect to the perception of the space in its entirety. This concept may in fact also be extended to the layout of furniture within an enclosure, though further empirical research in that direction can give us a more detailed insight into the dynamics of that field.

\section{Partition configurations and perceived de- gree of separation}

The set of enclosures pertaining to different configurations of partitions have been described in the previous section. The study was focused towards assessing perceived degree of separation that subjects attributed to each of these partition configurations. As was the case for the previous sets, the results obtained here did show a great degree of consistency across subjects. The inferences drawn out of this set are however more nuanced, and the pattern of responses seemed be less governed by any single predominant factor. The data indicates clearly that instance 4, i.e. the enclosure with a partition wall with clerestory opening, corresponds to the highest value of rated degree of separation, while instance 1 with the single vertical opening corresponds to the lowest value. (Figure 10) Again, the partition configuration with dense bars created a higher sense of separation as compared to the one with broader bars. The fact that instance 4 creates the greatest sense of separation is self-evident - there is no 'visual' connection between the two spaces that is presented to the subjects. This is in tune with the dominant inference drawn from the previous two sets - that of the major role of the visual field along horizontal eye level.

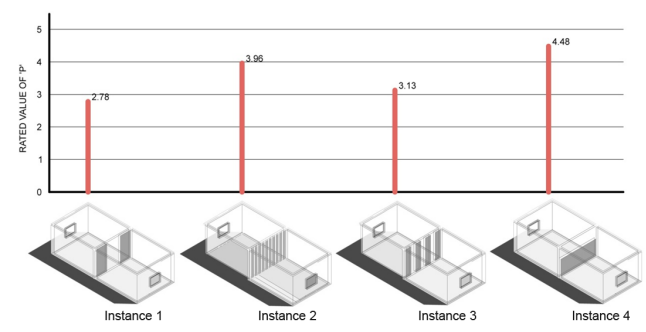

The underlying pattern (if any) behind the relative order of degrees of separation is not directly evident. The sole factor of openings along visual field at eye level alone cannot account for the nature of responses as all the variants except instance 4 have the exact same amount of opening along horizontal axis at eye level. (Figure 11)
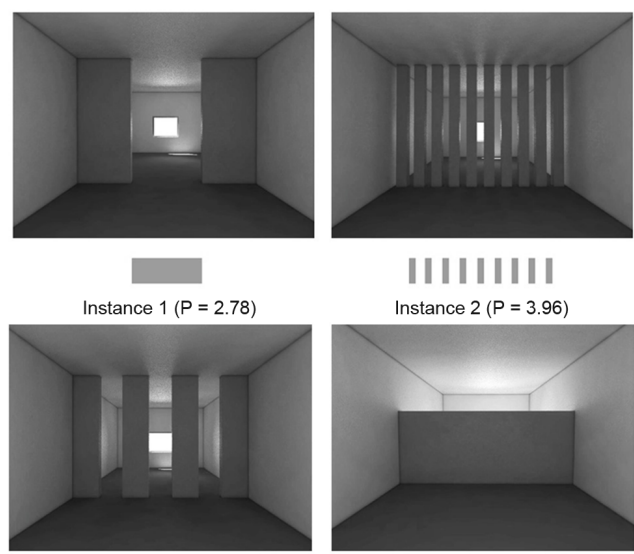

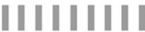

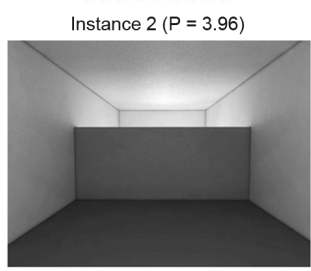

Instance $3(P=3.13)$

Instance $4(P=4.48$

There appears to be more than one defining factor here, many of which were hinted at through the running qualitative responses that were recorded. One such factor may be the issue of access. During briefing before experimentation, all subjects were asked to rate the degree of 'visual separation' between the two spaces that the partition provided, and were 
asked not to take possibility or ease of access into consideration. However, the two appear to be very closely linked from the perceptual point of view. Instance 1 presents the greatest ease of access between the spaces, and thus has a very low value in rated separation. Instance 3 again presents a possibility of access, and thus comes just above Instance 1 in ascending order of rated separation. For both Instance 2 and Instance 4, access was not a possibility. However Instance 2 presented a great degree of visual access between the two spaces, which was absent in Instance 4, thus explaining the relative order of rated separation. This analysis can be condensed to arrive at a more generalized factor that influences rated degree of separation. It may be said that larger unified openings along horizontal axis at eye level create a lower sense of separation as compared to smaller and more dispersed openings. This axiom may also pertain to access as larger unified openings automatically suggest a greater possibility of physical access.

This principle so derived can be used as a thumb rule and incorporated into the design process. The degree of visual connection between two spaces defines to a large extent one's experience in each of these spaces. A higher degree of connection allows for one space to exert a great deal of influence on how one will experience the other. This set of experiments has demonstrated that it is not only the amount opening, but the configuration of opening which also dictates the perceived degree of separation. The derived inferences can be employed by architects to create higher or lower degrees of perceived separation without changing the absolute amount of physical obstruction.

\section{CONCLUSION}

This this body of work was an initial step towards providing architects with a body of empirically validated data pertaining to the perceptual realms within space. This can, in due course of time, reduce the role of intuition in the design process, and lead to more rationally grounded design decisions pertaining to architectural experience. While some of the findings presented in this paper may already be in use in architectural practice as intuitive 'thumb rules', it only a scientific approach to the realm of perception that can allow us to uncover any underlying logic that governs our experiences. It is only when there is focused inquiry towards the nature of such correlations, that we can make use of our knowledge to achieve specific ends with respect to how we would want an occupant to perceive a space, possibly leading to the development of 'design standards' in the future with respect to perceptual attributes. The methodology adopted in this study has the potential to be drawn upon, refined, and applied in similar focused experiments, directed towards making the intangibles a little more tangible.

\section{REFERENCES}

Bitner, MJ 1992, 'Servicescapes: the impact of physical surroundings on customers and employees.', The Journal of Marketing, 56, pp. 57-71

Ching, FDK 1943, Architecture: Form, Space and Order, John Wiley and Sons

Franz, G, von der Heyde, M and Bulthoff, HH 2003 'An empirical approach to the experience of architectural space in VR', DIGITAL DESIGN - 22nd eCAADe Conference

Houtkamp, JM 2012, Affective appraisal of virtual environments, Gaming Research for Training and Environment

Kuller, R 1972, A semantic model for describing perceived environment, National Swedish Institute for Building Research, Stockholm

Mazuryk, T and Gervautz, M 2010, Virtual Reality: History, Applications, Technology and Future, Institute of Computer Graphics, Vienna University of Technology, Austria

Shemesh, A, Bar, M and Grobman, YJ 2015 'Space And Human Perception - Exploring Our Reaction to Different Geometries of Spaces', Emerging Experience in Past, Present and Future of Digital Architecture, Proceedings of the 20th International Conference of the Association for Computer-Aided Architectural Design Research in Asia CAADRIA 2015 